\title{
Quyu Shengji Formula Facilitates Diabetic Wound Healing via Inhibiting the Expression of Prostaglandin Transporter
}

\author{
Yi Lu, ${ }^{1}$ Xiaojie Ding, ${ }^{1}$ Fei Qi, ${ }^{2}$ Yi Ru, ${ }^{1}$ Le Kuai, ${ }^{1}$ Siting Chen, ${ }^{1}$ Yingyao Yang, ${ }^{1}$ Xin Li, \\ Fulun Li, ${ }^{1}$ Bin Li $\left(\mathbb{D},{ }^{1}\right.$ Mi Zhou $\left(\mathbb{D},{ }^{1}\right.$ and Kan Ze $\mathbb{D}^{1}$ \\ ${ }^{1}$ Department of Dermatology, Yueyang Hospital of Integrated Traditional Chinese and Western Medicine, \\ Shanghai University of Traditional Chinese Medicine/Institute of Dermatology, \\ Shanghai Academy of Traditional Chinese Medicine, Shanghai 201203, China \\ ${ }^{2}$ Department of Vascular, Shanghai Hospital of Integrated Traditional Chinese and Western Medicine, \\ Shanghai University of Traditional Chinese Medicine, Shanghai 200082, China \\ Correspondence should be addressed to Mi Zhou; vieky2866@163.com and Kan Ze; zekan1@163.com
}

Received 17 September 2020; Revised 25 December 2020; Accepted 13 January 2021; Published 23 January 2021

Academic Editor: Zihu Guo

Copyright (C) $2021 \mathrm{Yi} \mathrm{Lu}$ et al. This is an open access article distributed under the Creative Commons Attribution License, which permits unrestricted use, distribution, and reproduction in any medium, provided the original work is properly cited.

Background. Quyu Shengji Formula (QSF), a Chinese medicine formula widely used in the clinic, has proven therapeutic effects on diabetic ulcers. Nevertheless, the potential mechanism of how QSF cures diabetic ulcer remains elusive. Objective. To assess the mechanism of QSF against wound healing defects in diabetes. Methods. Db/db mice were adopted to determine the therapeutic potential of QSF. Further histology analysis was performed by hematoxylin and eosin (H\&E) staining. Moreover, the expression patterns of prostaglandin transporter (PGT), prostaglandin $\mathrm{E}_{2}\left(\mathrm{PGE}_{2}\right)$, and angiogenesis factor vascular endothelial growth factor (VEGF) were evaluated by immunostaining (IHC) analysis, ELISA assay, real-time quantitative polymerase chain reaction (RTqPCR), and western blot analysis in vivo. Human dermal microvascular endothelial cells (HDMECs) and the shRNA interference technique were used to explore the effects of QSF on cell migration, PGT, PGE ${ }_{2}$, and angiogenesis factor VEGF in vitro. Results. Applied QSF on the wound of db/db mice significantly accelerated wound closure. Reductions of PGT and elevations of PGE 2 and increased angiogenesis factor VEGF levels were shown after QSF treatment in vivo and in vitro. Furthermore, QSF promoted HDMEC migration. Inhibition of the expression of PGT by shRNA reversed phenotypes of QSF treatment in vitro. Conclusion. Taken together, our findings reveal that QSF ameliorates diabetes-associated wound healing defects by abolishing the expression of PGT.

\section{Introduction}

A diabetic ulcer is a serious complication of diabetes mellitus. Ulcers are often difficult to heal for a long time and easily lead to amputation and eventual disability. It has been reported that approximately $6.3 \%$ of the world's population suffers from diabetes [1]; moreover, a diabetic patient is found with amputation approaching every 30 seconds worldwide [2]. These facts highlight the urgency for scientific discovery-based therapeutic interventions [3].

Debridement and dressing change are often ineffective in treating diabetic ulcers and always cost a lot, which imposes a heavy body-heart burden on the patient, family, and even the whole society. However, some newly developed biological agents and new excipients are expensive or have poor efficacy, and some even pose an oncogenic risk [4]. Traditional Chinese medicine (TCM) has accumulated extensive experience in the repair and diagnosis of wound healing. Traditional experience has created several drugs based on the theory of "Qufu Shengji" (removing slough and promoting the growth of tissue regeneration). We summarized the clinical experience and found that "blood stasis" is also the important pathologic factors of diabetic ulcers. Based on the theory of "Quyu Shengji" (removing blood stasis and promoting the growth of tissue regeneration), we created the "Quyu Shengji" Formula (QSF). QSF has obtained a positive 
curative effect in the clinic and found that QSF plays a critical role in the regulation of collagen metabolism, MMP3, and Activin/Follistatin [5-8].

Prostaglandin $\mathrm{E}_{2}\left(\mathrm{PGE}_{2}\right)$ has a wide range of physiological functions, especially in the functions of dilating blood vessels and increasing blood flow. Recent studies showed that it has a considerable effect on the healing of gastric ulcers [9]. $\mathrm{PGE}_{2}$ level is decreased in chronic diabetic ulcer, which may be an essential reason why diabetic ulcer is difficult to heal [10]. However, the underlying mechanisms remain unclear. $\mathrm{PGE}_{2}$ is synthesized by cyclooxygenase (COX) and transported by prostaglandin transporter (PGT) to intracellular degradation. Recent research showed that COX increases in diabetic ulcer wounds [11]. Notably, the reason for the decrease of $\mathrm{PGE}_{2}$ can only be analyzed from the perspective of metabolism.

PGT was found relatively late, and its research is still at a relatively preliminary stage. PGT and $\mathrm{PGE}_{2}$ showed an obvious negative correlation, and reducing PGT could increase the content of PGE [12], and vice versa. It plays an important role in fever, pregnancy, and acromegaly [13-15]. Studies have revealed that the increased expression of PGT in chronic diabetic ulcers contributes to increased degradation of $\mathrm{PGE}_{2}$, leading to a decrease in local $\mathrm{PGE}_{2}$ content, which is a crucial reason for delayed wound healing. Inhibition of PGT can facilitate wound healing of diabetic ulcers [12].

Here, we created QSF based on the theory of "Quyu Shengji." "Quyu" means "removing blood stasis." In diabetic chronic skin ulcers, the increase in PGT causes a decrease in $\mathrm{PGE}_{2}$, leading to local occlusion of blood vessels similar to what TCM calls "Yu" (blood stasis). We adopted $\mathrm{db} / \mathrm{db}$ mice and human microvascular endothelial cells (HDMECs) as the research object to evaluate QSF regulating the expression of PGT during diabetic healing time and its effects on the metabolism of $\mathrm{PGE}_{2}$ and VEGF.

\section{Materials and Methods}

\subsection{Plant Material}

Drug Preparation. QSF was comprised of 8 Chinese herbs, as shown in Table 1 . The dosage used in the present study was determined according to the Chinese Pharmacopoeia (2015 edition). The herbs were obtained from the pharmacy department of Yueyang Hospital of Integrated Traditional Chinese and Western Medicine, Shanghai University of Traditional Chinese Medicine.

The original medicinal materials were decocted twice in water according to the conventional method of decocting traditional Chinese medicine, then filtered and concentrated into $10 \mathrm{~g} / \mathrm{ml}$ medicinal solution, added with $70 \%$ ethanol for precipitation, and left for 24 hours. After the ethanol is recovered, it is diluted with an appropriate amount of water to make a mixture and finally made into a crude drug-containing $2 \mathrm{~g} / \mathrm{ml}$ [16]. All the pharmaceutical processes are prepared by the Pharmaceutical Preparation Room of Yueyang Hospital of Integrated Traditional Chinese and Western Medicine Affiliated with Shanghai University of Traditional Chinese Medicine.
2.2. Animals. Female clean-grade $\mathrm{db} / \mathrm{db}$ mice and C57 mice, weighing $20 \pm 4 \mathrm{~g}$, were provided by the Experimental Animal Center of the Chinese Academy of Sciences, Shanghai. Animals were maintained under specific pathogen-free conditions. All experiments were performed according to the guidelines of the Committee on Protection, Welfare and Ethics of Experimental Animals in Yueyang Hospital of Integrated Traditional Chinese and Western Medicine affiliated to Shanghai University of Traditional Chinese Medicine (No. YYLAC-2019-043).

2.3. Wound Model. All mice were anesthetized by intraperitoneal injection of $0.1 \%$ ketamine hydrochloride injection $(100 \mathrm{mg} / \mathrm{kg})$. The modeling area was marked with a stamp with a diameter of $8 \mathrm{~mm}$, and both sides of the back spine were shaved with a mechanical razor. To form an open wound with a full-thickness skin defect with a diameter of $8 \mathrm{~mm}$ under strict aseptic operation, the left and right sides of the mouse spine were cut to the subcutaneous fascia layer. There are 4 mouse wound models with skin ulcers on the left and right sides [17].

2.4. Animal Grouping and Administration Method. After adaptive feeding for 1 week, 12 mice were randomly divided into 3 groups as follows: blank group, control group, and QSF group. The adult dose of QSF was $130 \mathrm{~g}$ per day according to the adult standard weight of $70 \mathrm{~kg}$, the mouse coefficient was 9 , and the daily dose of rats was $16.7 \mu \mathrm{g} / \mathrm{g}$. An equal volume of distilled water was administered by gavage in the blank and control groups. The intragastric volume was calculated by $2 \mathrm{~g} / \mathrm{ml}$ once a day.

We repeated the experiment three times, sacrificing animals on day 1 , day 5 , and day 9 , respectively.

2.5. Macroscopic Evaluation. The measurement of the wound area was performed as follows: the quick wound healing adhesive plaster was pressed to the ulceration surface, and a marking pen was used to draw the outline of the wound on the adhesive plaster. A photo of the plaster was taken using a digital camera against a white background. Wound area measurement: then the photo was evaluated by calculating the wound area with image analysis software

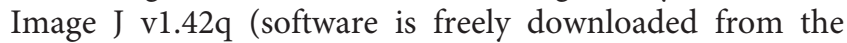
National Medical Center website). The wound area on day 1 , day 3 , day 5 , and day 9 after modeling was compared.

2.6. He E and Immunochemistry. On day 1 , day 5 , and day 9 after the wounding, animals were euthanized by $\mathrm{CO}_{2}$ and the tissues of the skin wounds were taken with an $8 \mathrm{~mm}$ diameter metallic punch. The tissue was put into liquid nitrogen immediately and kept at $-80^{\circ} \mathrm{C}$.

$\mathrm{HE}$ and immunohistochemical staining were carried out according to the relevant protocols. For general histological analyses, tissue samples were fixed in $10 \%$ neutral-buffered formalin, embedded in paraffin, sectioned from the midline of wounds, and stained with H\&E. 
TABLE 1: Ingredients of QSF with English translations.

\begin{tabular}{|c|c|c|c|}
\hline Main composition & Latin scientific name & Plant part (s) & Amount (g) \\
\hline radix astragali & Astragalus membranaceus (Fisch.) Bge. var. mongholicus (Bge.) Hsiao & Root & 30 \\
\hline Radix pseudostellariae & Pseudostellaria heterophylla (Miq.) Pax et Pax et Hoffm. & Root & 9 \\
\hline Rhizoma atractylodis macrocephalae & Atractylodes macrocephala Koidz & Root & 9 \\
\hline Radix Rehmannia & Rehmannia glutinosa Libosch. & Root & 12 \\
\hline Radix salviae miltiorrhizae & Salvia miltiorrhiza Bge. & Root & 30 \\
\hline Hirudo & Whitmania pigra Whitman & All & 6 \\
\hline Semen Persicae & Prunus persica (L.) Batsch & Seed & 9 \\
\hline Rhizoma chuanxiong & Ligusticum chuanxiong Hort. & Root & 9 \\
\hline
\end{tabular}

The expression of PGT in wound tissues was determined with immunohistochemical analysis and the following antibodies: rabbit anti-PGT (diluted: 1:150; all from BioTNT, Shanghai, China), and the secondary antibodies goat antirabbit IgG HRP (diluted: 1:2500). Under the same experimental conditions as the negative control, each sample was diluted and incubated with the same type of antibody. Images were acquired using a light microscope (Olympus, Tokyo, Japan) and analyzed with the image processing software (Image-Pro Plus 6.0).

IHC average optical density value analysis method: each slice in each group was randomly selected with at least $3200 \mathrm{x}$ fields of view for photography. The same brown color was selected as the unified criterion for judging the positivity of all photos by using Image-Pro Plus 6.0. The cumulative optical density value (IOD) and the pixel area (AREA) of each photo were obtained by analyzing each photo. Calculate the average optical density value ( $\mathrm{AO}$ value); $\mathrm{AO}=\mathrm{IOD} /$ AREA; the higher the $\mathrm{AO}$ value, the higher the positive expression level.

2.7. RT- $q P C R$. The sampling procedure is the same as above. Extracting total RNA: the homogenate was taken and total RNA was extracted according to the Trizol reagent kit method and steps (Invitrogen, Carlsbad, CA, USA). The concentration of total RNA was measured by ultraviolet spectrophotometer. The purity of RNA was measured with agarose gel electrophoresis. Reverse transcribed to synthesize cDNA, primers used were as Table 2.

Reverse Transcription System First Strand cDNA Synthesis Kit was used, with a total reaction volume of $20.0 \mu \mathrm{l}$. Real-time PCR amplification: SYBR Green kit (Thermo, Eugene, OR, USA) was used to make real-time PCR amplification of GAPDH, PGT, and VEGF. Real-time fluorescent PCR was used to make a real-time fluorescent quantitative PCR reaction (Applied Biosystems, Foster City, CA, USA). After the reaction was finished, fluorescent quantitative data were collected and analyzed. The data included the amplification curve, working curve, dissociation curve, and corresponding CT value. Light Cycler Software (Version 3.5) was used for data analysis.

2.8. ELISA. The levels of $\mathrm{PGE}_{2}$ in wound tissue or cell supernatant were determined using ELISA (sensitivity $0.8-300 \mathrm{pg} / \mathrm{mL}$; R\&D Systems, Minneapolis, MN, USA; ELISA kits were from BioTNT, Shanghai, China) according to the manufacturer's instructions. Set the blank to zero and measure the absorbance (OD value) of each hole in sequence at $450 \mathrm{~nm}$ wavelength.

2.9. Western Blot. The tissues were lysed and the supernatant was used for protein quantification. GAPDH was used as a protein loading control. The following primary antibodies were used in subsequent experiments: antibody PGT (from Biorbyt, UK) and VEGF antibody (from Abcam, Cambridge, Massachusetts, USA). The secondary antibodies used are goat anti-rabbit HRP and goat anti-mouse HRP (from Biyuntian, Shanghai, China). A Tanon-5200 imaging system (Tanon Science and Technology Co., Shanghai, China) was used to check the target protein and was observed with autoradiography film.

2.10. Drug Serum. Male clean-grade SD rats, weighing $250 \pm 20 \mathrm{~g}$, were provided by the Experimental Animal Center of the Chinese Academy of Sciences, Shanghai. Animals were maintained under specific pathogen-free conditions. When QSF was given by gavage for 7 days continuously, the rats were killed by $\mathrm{CO}_{2}$. Blood samples were taken from the heart aseptically. Samples were left at room temperature for 3-4 hours. They were centrifuged for $10 \mathrm{~min}$ at 3000 revolutions/min. Then, we took the supernatant, and it was inactivated in a water bath at $56^{\circ} \mathrm{C}$ for 30 minutes. We filtered the serum through a $22 \mu \mathrm{m}$ filter membrane in an ultraclean table to remove bacteria. Serum samples were dispensed into autoclaved centrifuge tubes. The drug serum was stored at $-20^{\circ} \mathrm{C}[18]$.

2.11. Cell Culture and Administration Method. Human dermal microvascular endothelial cells (HDMECs) were purchased from Kilton Biotechnology (Shanghai) Co., Ltd. The experiment is grouped as follows:

(a) Low glucose (blank) group: $5 \mathrm{mmol} / \mathrm{L}$ glucose $+10 \%$ blank serum

(b) High glucose (control) group: $25 \mathrm{mmol} / \mathrm{L}$ glucose $+10 \%$ blank serum

(c) QSF prescription medicine serum group: $25 \mathrm{mmol} / \mathrm{L}$ glucose $+10 \%$ QSF drug serum

HDMECs were cultured in endothelial cell medium (ECM) containing $5 \%$ fetal bovine serum, $1 \%$ endothelial cell 
TABle 2: Primers.

\begin{tabular}{lccc}
\hline Species & Target & Direction & Sequence \\
\hline \multirow{2}{*}{ Mouse } & \multirow{2}{*}{ PGT } & Forward & 5everse \\
\hline \multirow{2}{*}{ Mouse } & \multirow{2}{*}{ VEGF } & Forward & 5-TCGATAGTGGTGAGGCTGCT-3 \\
& & Reverse & 5-AATGCTCGGTCTTCAACAACAT-3 \\
\hline \multirow{2}{*}{ Mouse } & \multirow{2}{*}{ GAPDH } & Forward & 5-GCTTCCTACAGCACAGCAGA-3 \\
& & Reverse & 5-GGGCATCTTGGGCTACACTG-3 \\
& & 5-CATGAGGTCCACCACCCTGT-3 \\
\hline
\end{tabular}

growth supplement (ScienCell Research Laboratories), 1\% penicillin-streptomycin, and 5 or $25 \mathrm{mmol} / \mathrm{L}$ glucose.

Each group had 4 wells; each group was seeded in the corresponding glucose environment at $37^{\circ} \mathrm{C}$ for 3 days. Medicated serum was added and cultured for 12 hours and then digested with trypsin. Cells were collected for further experiment.

2.12. Establishment of the Lentiviral Vector Carrying PGT shRNA. We completed the design of the target gene interference target and then sent it to the gene synthesis company (Kilton Biotechnology Co., Ltd. Shanghai, China) to synthesize the interference target shRNA as Table 3 and completed the shRNA double-strand annealing after synthesis. The vector was linearized and recovered, connected with the annealed shRNA, and transformed by the electrochemical method to complete the identification of positive clones. Recombination interferes with lentivirus packaging according to the requirements of the kit. The transfection experiment was completed, and the cell pellet was collected 48 hours after transfection for PCR experiment to screen the sites with better interference effect. PCR identifies the shRNA with the best interference efficiency and then uses this shRNA for subsequent experiments. Based on the front result, they were divided into a blank group (high glucose, no intervention), shPGT group (high glucose + shPGT interference, interference group), QSF group (high glucose + QSF drug serum), and shPGT + QSF group (high glucose + shPGT interference+QSF drug serum).

2.13. Transwell. Briefly, we infiltrate the chamber before inoculation. We put the Transwell chamber into the culture plate, prepared the cell suspension, and inoculated the cells. Cells were fixed at room temperature and observed after staining.

2.14. Statistical Analysis. GraphPad Prism 8 (GraphPad Inc., CA, USA) software was used for statistical analysis. The measurement data was expressed by $x \pm \mathrm{SD}$, and the count data was expressed by the rate. If multiple groups of measurement data conform to the normal distribution (ShapiroWilk, SW method) and the variances were uniform, the analysis of variance and Tukey's method for pairwise comparison would be applied. The nonparametric test was used for those who do not meet the conditions of the variance test and Mann-Whitney U method. The Kruskal-
Wallis method was applied for the comparison of multiple groups, and the Dunnett method was used for the comparison between groups.

\section{Results}

3.1. QSF Promoted Diabetic Wound Healing in $d b / d b$ Mice. To test if QSF can overcome wound healing defects seen in $\mathrm{db} / \mathrm{db}$ mice, we examined the topical application of QSF in vivo. From Figures 1(a) and 1(c), it was obvious that wound healing in the control group composed of $\mathrm{db} / \mathrm{db}$ mice was more difficult than that that in the blank group with normal blood glucose, while wound healing in the QSF group was better than that in the control group. In particular, wound healing accelerated on day 5 after wounding. Histologically, it was obvious that the infiltration of inflammatory cells in the QSF group was less than that in the control group, while the tissue growth and reepithelialization of QSF were superior to those in the control group (Figure 1(b)). Therefore, from a superficial point of view, we found that QSF could promote wound healing in $\mathrm{db} / \mathrm{db}$ mice. The effect of QSF on promoting the healing of diabetic wound model was accelerated with the progress of time, which was obvious in the middle and late stages of wound healing.

3.2. QSF Inhibited the Expression of PGT and Upregulated $P G E_{2}$ and VEGF in Wound Healing of $d b / d b$ Mice. Next, we examined whether the observed accelerated wound closure of QSF wounds could, at least in part, contribute to the inhibition of PGT and the upregulation of $\mathrm{PGE}_{2}$ and VEGF. We detected the expression of the molecules mentioned above. As shown in Figure 2(c), compared with the blank group, $\mathrm{PGE}_{2}$ content in the control group was significantly decreased, while that in the QSF group increased $\mathrm{PGE}_{2}$ content. Such an effect even appeared in the middle stage of wound healing.

From Figures 2(a), 2(b), 2(d), and 3, IHC, PCR, and WB were used to verify that in the $\mathrm{db} / \mathrm{db}$ mouse ulcer model, tissue PGT expression increased significantly, while VEGF expression decreased. The QSF group inhibited the high expression of PGT and increased the expression of VEGF, and this effect began to occur as early as the middle stage of wound healing.

3.3. QSF Improved the Migration Ability of HDMEC and Regulated $P G E_{2}, P G T$, and VEGF. Due to HDMEC's ability to improve local angiogenesis of the wound and enhancing blood supply, we examined whether QSF affects the 
TABLE 3: shRNA design sequence.

\begin{tabular}{lr}
\hline PGT locus 1 (370-388) & GGTGTTTGTGCTCTGCCAA \\
\hline PGT-F & CCGGTGGTGTTTGTGCTCTGCCAACTCGAGTTGGCAGAGCACAAACACCTTTTTG \\
\hline PGT-R & AATTCAAAAAGGTGTTTGTGCTCTGCCAACTCGAGTTGGCAGAGCACAAACACCA \\
\hline PGT locus 2 (444-462) & CCACCATTGAGAAGCGCTT \\
\hline PGT-F & CCGGTCCACCATTGAGAAGCGCTTCTCGAGAAGCGCTTCTCAATGGTGGTTTTTG \\
\hline PGT-R & AATTCAAAAACCACCATTGAGAAGCGCTTCTCGAGAAGCGCTTCTCAATGGTGGA \\
\hline PGT locus 3 (1998-2016) & CCATTGACCACTCCTGCAT \\
\hline PGT-F & CCGGTCCATTGACCACTCCTGCATCTCGAGATGCAGGAGTGGTCAATGGTTTTTG \\
\hline PGT-R & AATTCAAAAACCATTGACCACTCCTGCATCTCGAGATGCAGGAGTGGTCAATGGA \\
\hline
\end{tabular}

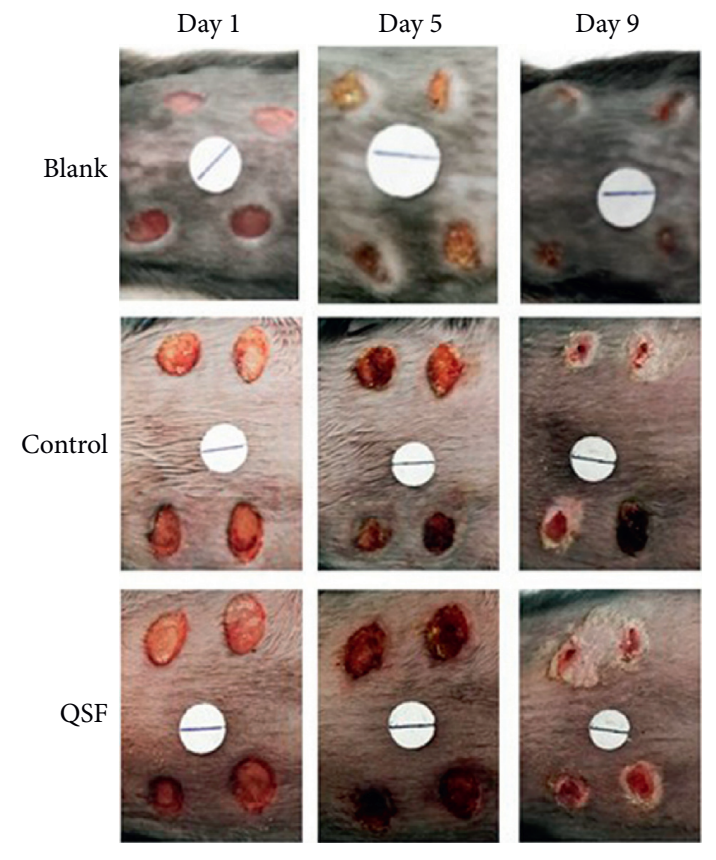

(a)

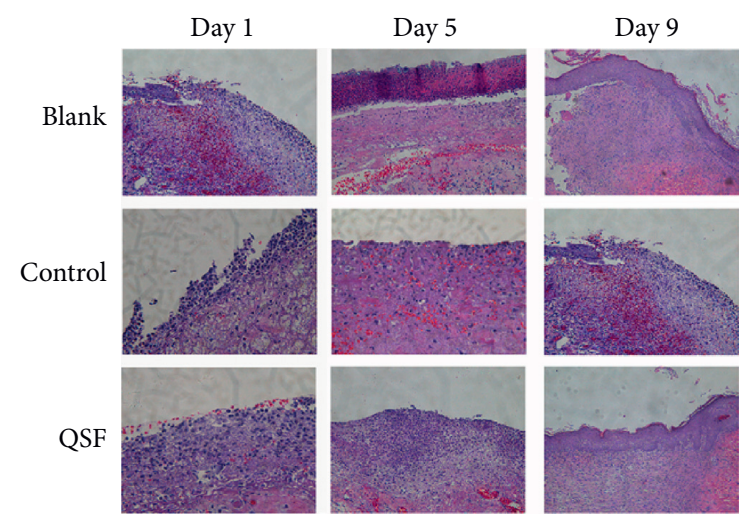

(b)

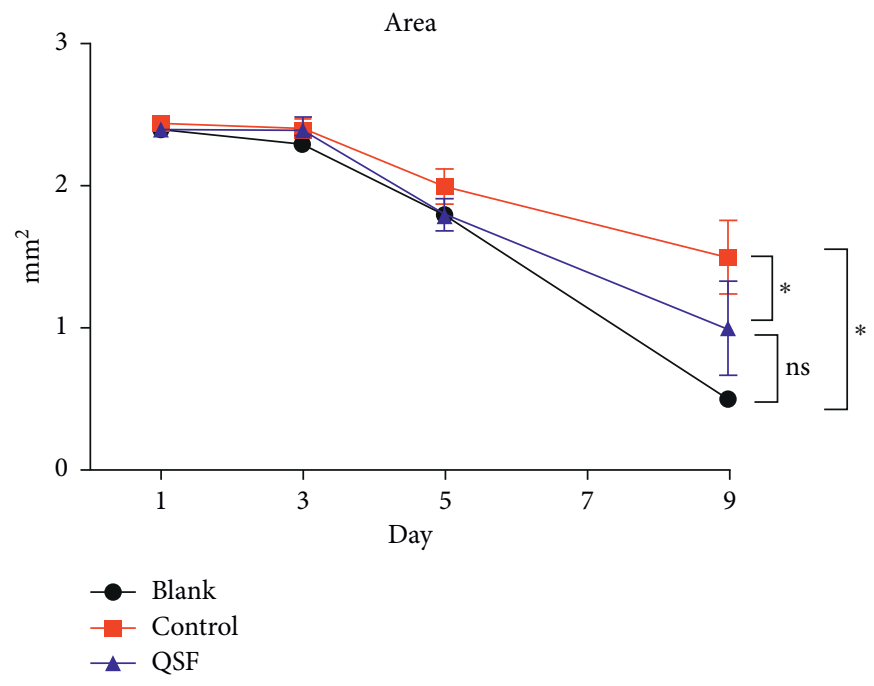

(c)

FIGURE 1: (a) Photographic representation of wound closure on different postwounding days in the blank, control, and QSF groups. Wounding days start from day 1, day 5, and day 9, respectively. (b) Wounded skin by H\&E staining in the same groups. Scale $=100 \mu \mathrm{m}$. (c) Wound area in the blank, control, and QSF groups from day 1, day 3, day 5, and day 9. ${ }^{*} P<0.05$, blank vs QSF and control. 

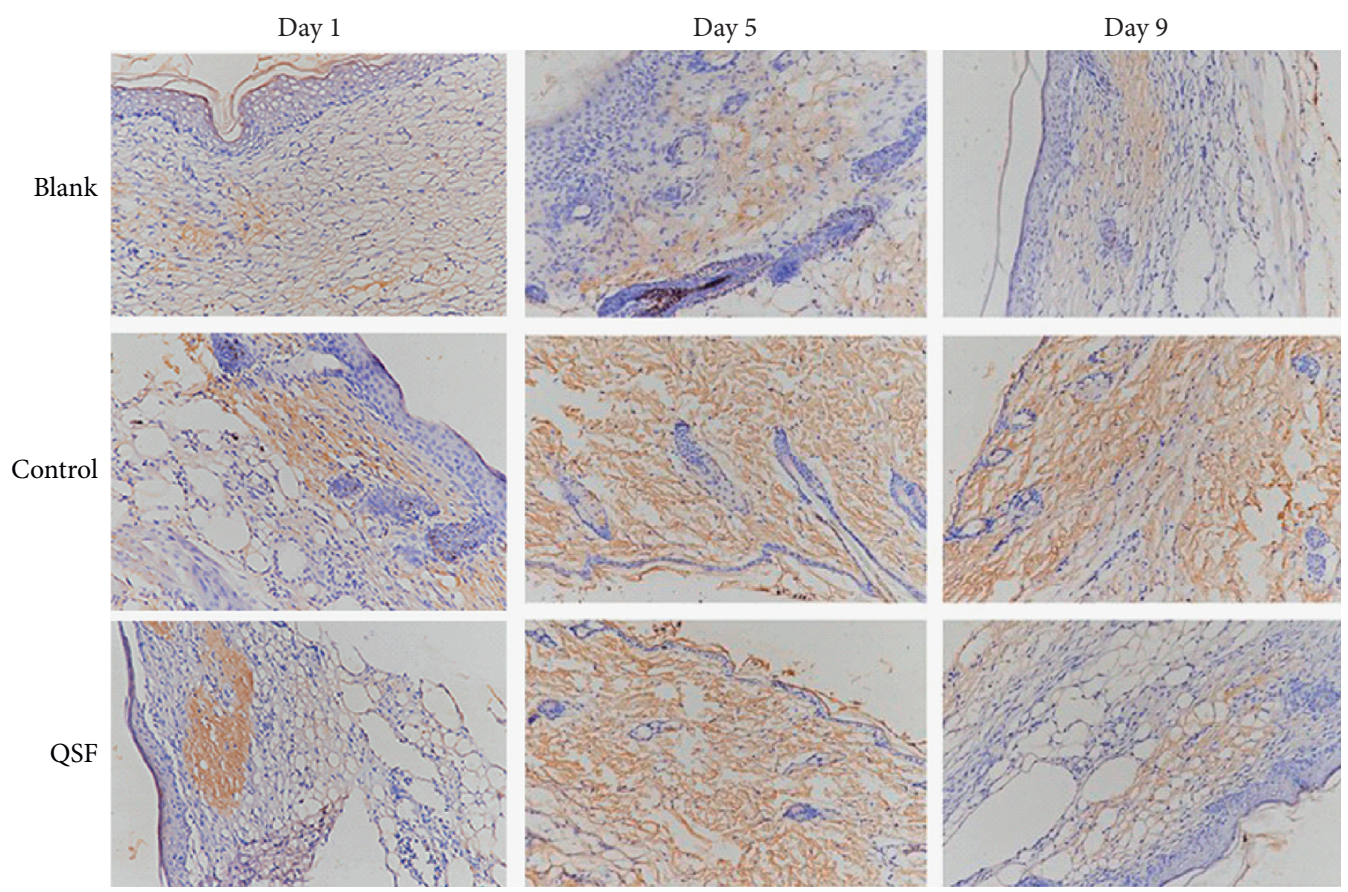

(a)
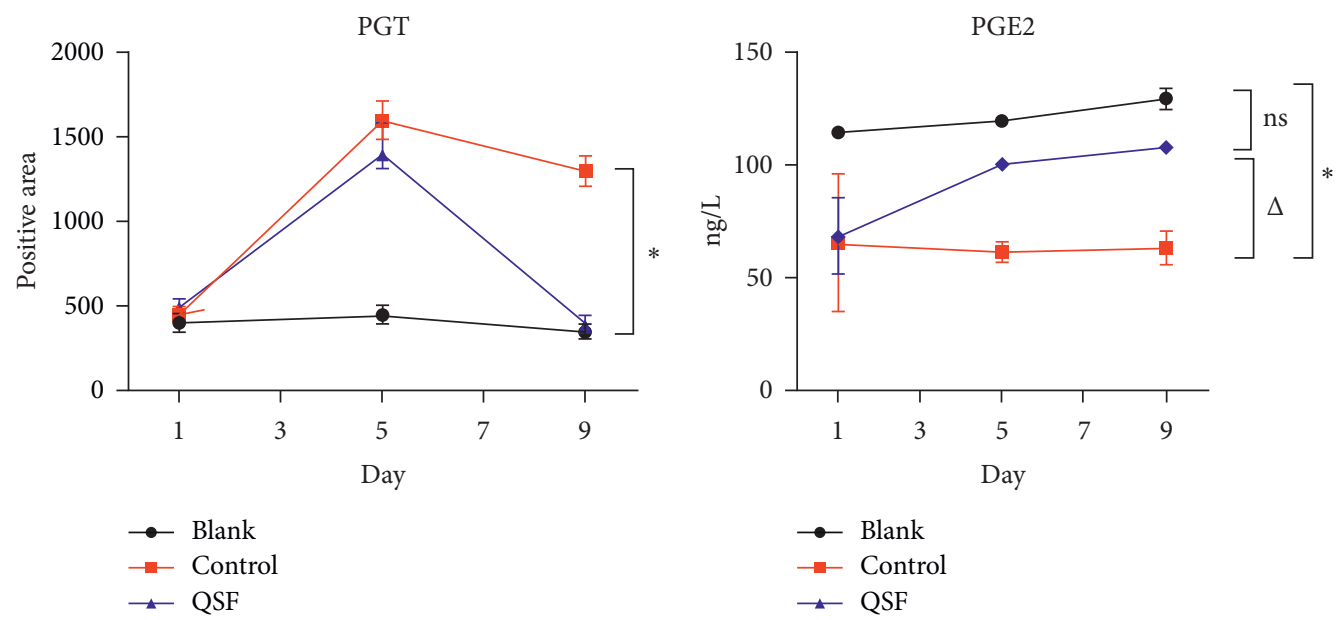

(b)

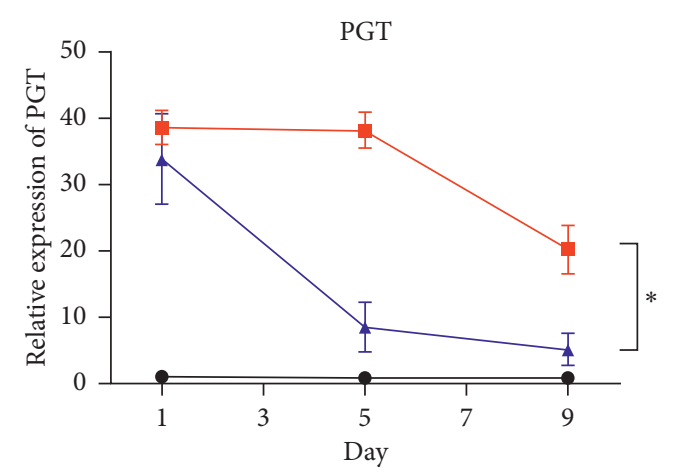

(c)

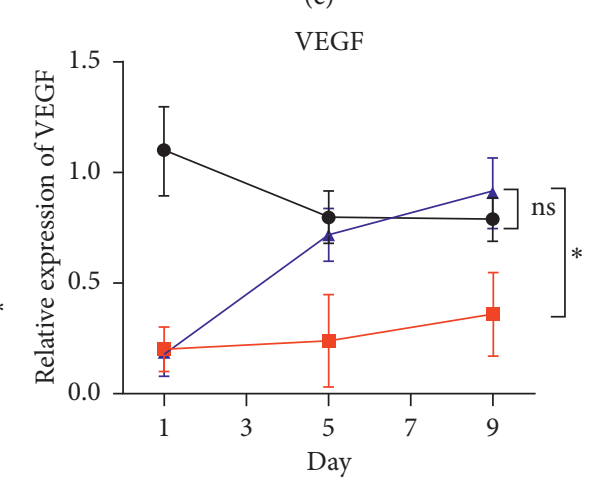

$$
\begin{aligned}
& \rightarrow \text { Blank } \\
& - \text { Control } \\
& \longleftarrow \text { QSF }
\end{aligned}
$$

(d)

FIGURE 2: (a) The pictures of immunochemistry of PGT in the blank, control, and QSF groups of day 1, day 5, and day 9. Scale $=100 \mu \mathrm{m}$. (b) Positive area of PGT in the same groups of day 1 , day 5 , and day 9. ${ }^{*} P<0.05$, control vs QSF and blank. (c) The concentration of PGE 2 in the same groups of day 1 , day 5 , and day $9 .{ }^{*} P<0.05$, control vs QSF. (d) Relative expression of PGT and VEGF in the same groups of day 1 , day 5, and day 9. ${ }^{*} P<0.05$, control vs QSF. 


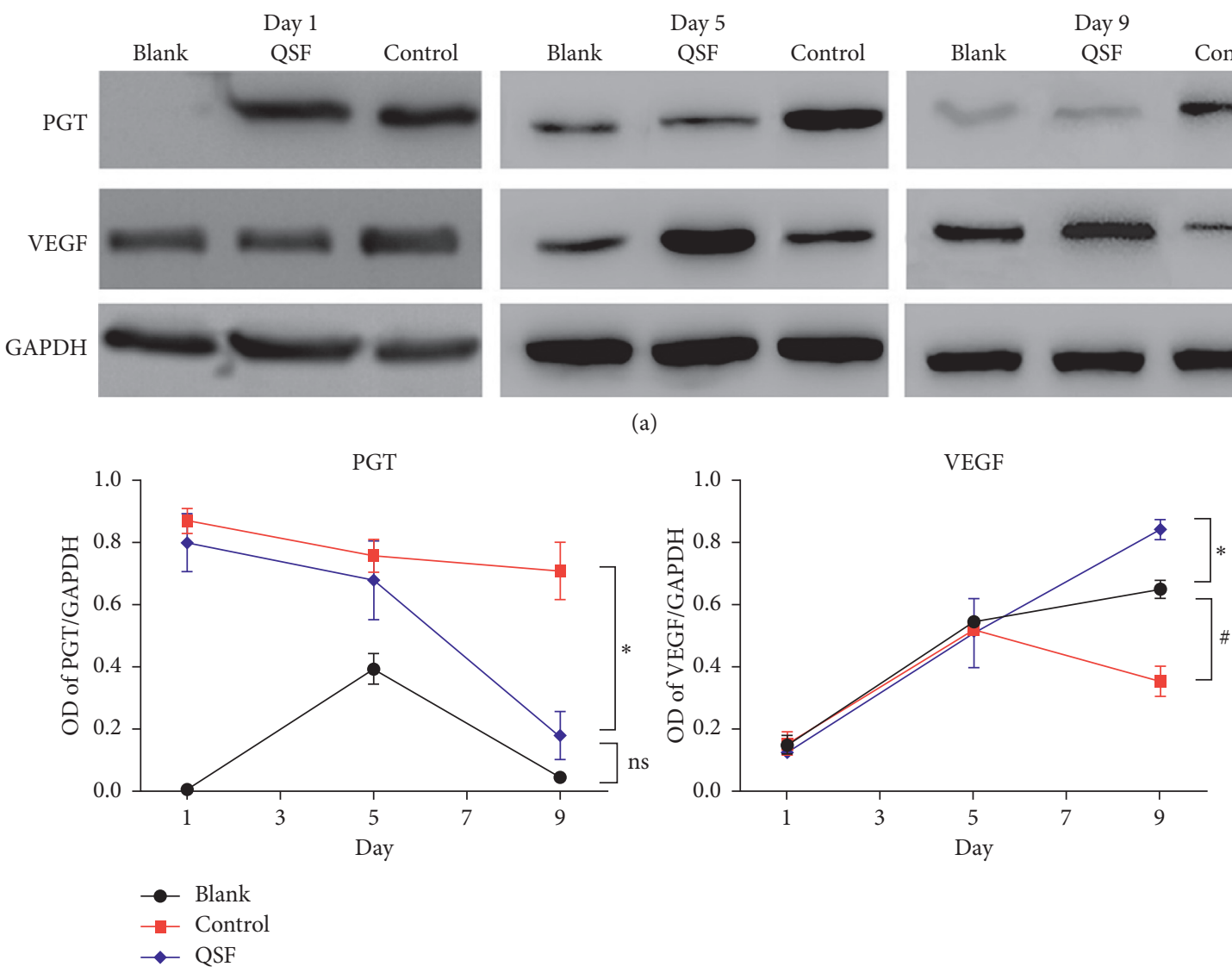

(b)

FIGURE 3: (a) Protein level of PGT and VEGF by western blot in the blank, control, and QSF groups of day1, day 5, and day 9. (b) OD of PGT/ GAPDH and VEGF/GAPDH in the same groups. ${ }^{*} P<0.05$, control vs. QSF. $\# P<0.05$ control vs blank.
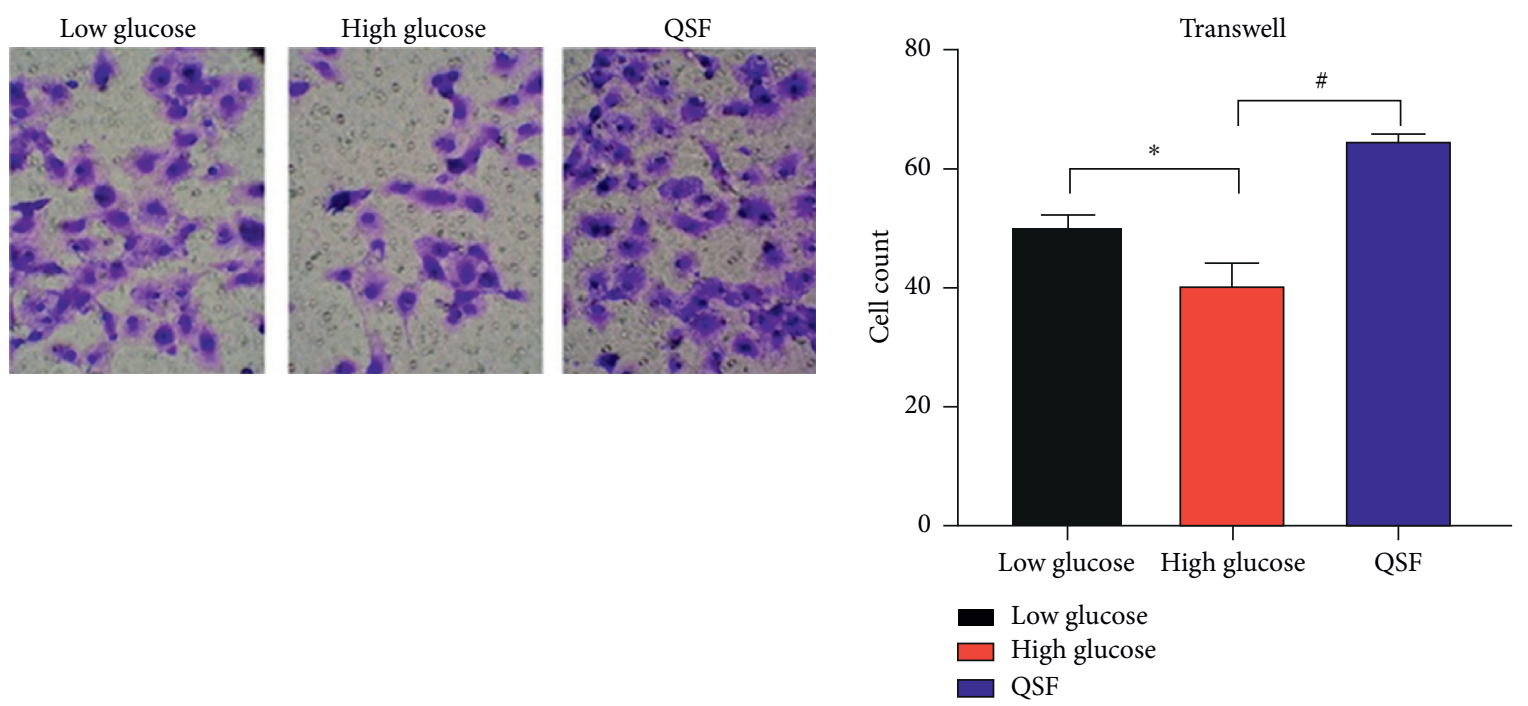

(a)

(b)

FIgURE 4: Continued. 


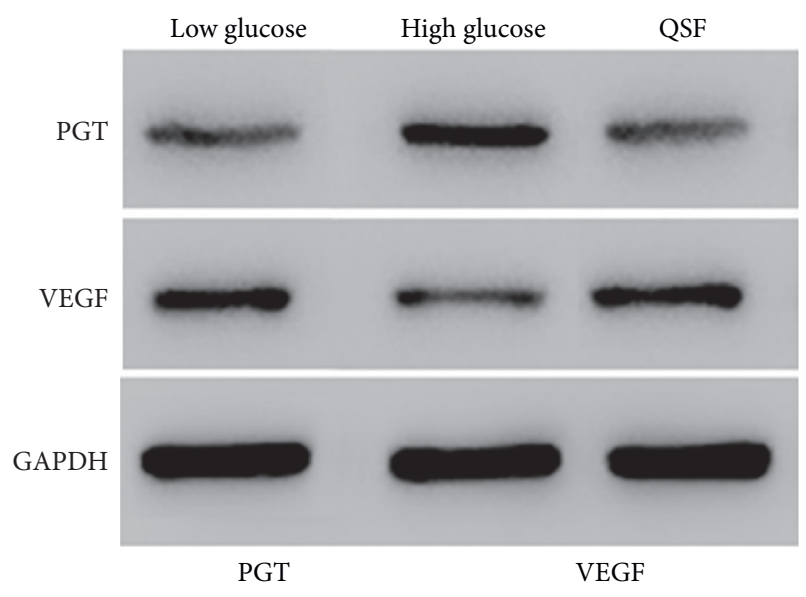

(c)

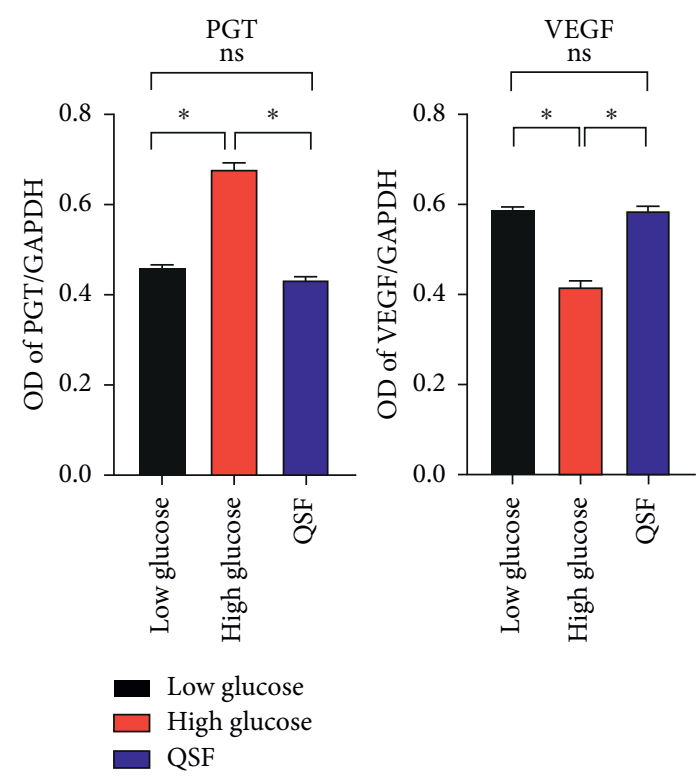

(d)

Figure 4: (a) The pictures of Transwell in the low glucose, high glucose, and QSF groups. (b) Cell count in the same groups, ${ }^{*} P<0.05$, high glucose vs QSF and low glucose. (c) The protein level of PGT and VEGF in the same groups. (d) OD of PGT/GAPDH and VEGF/GAPDH in the same groups. ${ }^{*} \mathrm{P}<0.05$, high glucose vs QSF and low glucose.

migration ability of HDMEC. Through Figures 4(a) and 4(b), we found that the migration capacity of HDMECs under high-glucose environment feeding was reduced, and this result could be corrected by QSF. Meanwhile, the protein expression of PGT in the high-glucose environment was significantly higher than that in the low-glucose environment, while the expression of VEGF was significantly decreased. QSF reduced the PGT expression of HDMEC and improved VEGF in the high-glucose environment (Figures 4(c) and 4(d)).

3.4. The Ability ofQSF on Cell Migration and Regulation $P G E_{2}$, $P G T$, and VEGF Was Decreased after PGT Was Blocked. Three shRNA sequences were used for the validation test. It was found in Figure 5(a) that empty vector had no effect on PGT expression, while shPGT-2 had the best inhibitory efficiency, so we chose shPGT-2 for subsequent study.

By the ELISA test, we found that after shPGT inhibited the expression of PGT, the content of $\mathrm{PGE}_{2}$ increased significantly, and the QSF group also achieved similar results. However, if shPGT inhibited the expression of PGT, QSF did not play a role as before, indicating that the effectiveness of QSF depended on PGT to a certain extent. Interestingly, similar results were obtained in the Transwell test in the same situation, which also indicates that the effect QSF group is to some extent dependent on the expression of PGT.

\section{Discussion}

Prostaglandin transporter (PGT) is the main protein that mediates the transmembrane transport of PGs such as $\mathrm{PGE}_{2}$. It is difficult for PGs to pass through the cell membrane in a freely diffused manner $[14,19]$. Therefore, PGT mediates the transmembrane transport of PG through the lactate-PG transport mechanism and is the main transport carrier of PG. PGT can transport PGs outside the cell membrane into the cell membrane, thereby inactivating PGs outside the cell membrane [20]. Previous studies have found that for the extracellular environment, increasing the glucose concentration will reduce $\mathrm{PGE}_{2}$, while decreasing the glucose concentration will increase $\mathrm{PGE}_{2}$ [21].

In recent years, studies on diabetic ulcer wounds have shown that increased tissue glucose content will cause PGTmediated reduction of $\mathrm{PGE}_{2}$ content, leading to a series of pathological changes such as local tissue capillary occlusion and local tissue hypoxia [22, 23].

$\mathrm{PGE}_{2}$ is transferred into the cell through the intercellular PG transporter and is rapidly inactivated by 15 -ketoprostaglandin (15-PGDH). In diabetic animals, $\mathrm{PGE}_{2}$ synthase did not decrease, but the expression of PGE2 decreased, which made the wound difficult to heal. High glucose levels will increase PGT and accelerate $\mathrm{PGE}_{2}$ metabolism. PGT inhibitors can accelerate wound healing and increase VEGF expression $[24,25]$.

The project that the author participated in the early stage partly clarified the mechanism of TCM on wound healing from the perspectives of the "stasis-removing and tissuegenerating" method regulating growth factors, collagen metabolism, MAPK, and Wnt/ $\beta$-catenin signaling pathways $[5-8,17]$. In the process of clinical diagnosis and treatment, the improvement of QSF on wound healing of diabetic patients has been fully affirmed by patients, but compared with the complex human environment, there are still many shortcomings in cell-level experiments. TCM also improves 

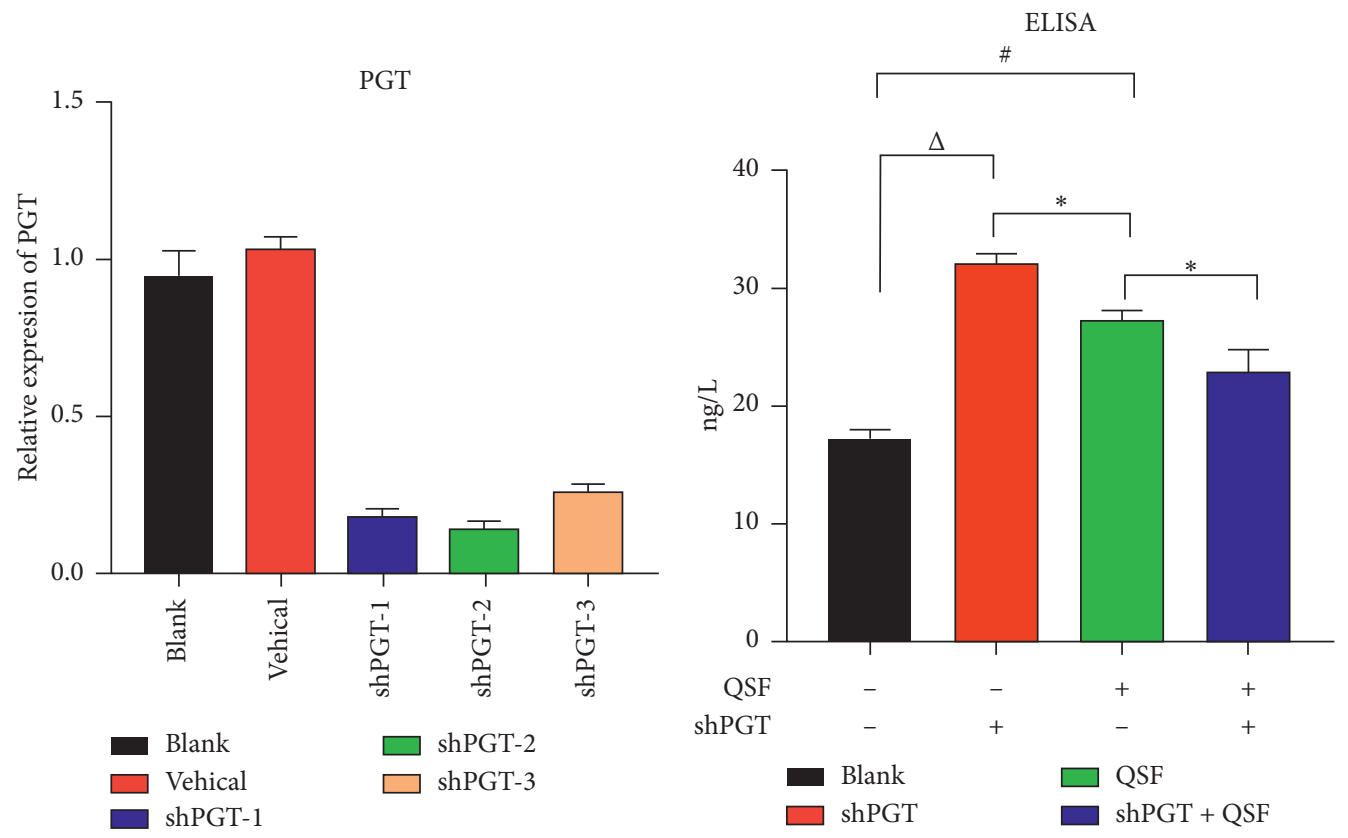

(a)
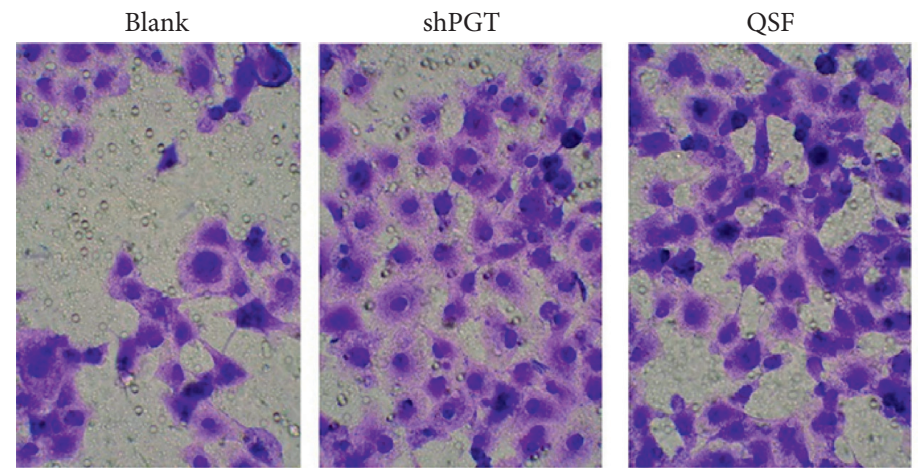

(b)

(c)

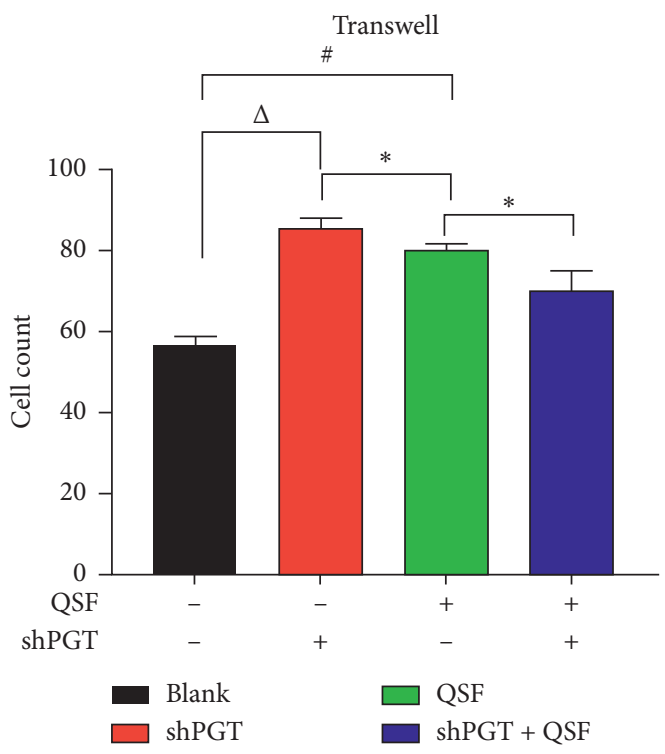

(d)

FIGURe 5: (a) Relative expression of PGT in the blank, vehicle, shRNA-PGT-1, shRNA-PGT-2, and shRNA-PGT-3 groups. (b) The concentration of $\mathrm{PGE}_{2}$ in the blank, shRNA-PGT, QSF, and shRNA-PGT combined with QSF groups. ${ }^{*} P<0.05$, QSF group vs shRNA + QSF and shRNA group; $\# P<0.05$, QSF vs blank; $\Delta P<0.05$, shPGT group vs blank group. (c) The pictures of Transwell in the same groups. (d) Cell count in the same groups. ${ }^{*} P<0.05$, QSF group vs shRNA + QSF group; $\# P<0.05$, QSF vs blank; $\Delta P<0.05$, shPGT group vs blank group. 
wound healing in multiple ways and targets. To further improve the theory of removing blood stasis and promoting muscle growth, more detailed cell pathway exploration is needed.

We created QSF based on the theory of "Quyu Shengji." "Quyu" means "removing blood stasis." In diabetic chronic skin ulcers, the increase in PGT causes a decrease in PGE2, leading to local occlusion of blood vessels similar to what TCM calls "Yu" (blood stasis). The theory of "Quyu" (removing blood stasis) is one of the main basic theories of TCM. In particular, great progress has been made in the past 200 years, making certain new treatment methods available for diseases that were difficult to treat in the past [26-29].

In the current study, $\mathrm{db} / \mathrm{db}$ mouse skin ulcer model was used as the research object. It was found that, compared with the blank group, the control group had difficulty in wound healing, impaired local blood supply, decreased tissue $\mathrm{PGE}_{2}$ and VEGF expression, and increased PGT expression. The traditional Chinese medicine QSF Decoction improves the series of pathological changes by inhibiting the expression of $\mathrm{PGT}$ and increasing $\mathrm{PGE}_{2}$ and VEGF in vivo and in vitro.

\section{Conclusion}

In summary, QSF attenuated PGT expression which promoted diabetic wound healing. Further studies on the effects of PGT knockout might reveal more molecular roles for PGT in diabetic wound healing. Our future direction is to further investigate the effect of PGT on nonhealing wounds.

\section{Data Availability}

The data used to support the findings of this study were supplied by Dr. Kan Ze under license and so cannot be made freely available. Requests for access to these data should be made to Dr. Kan Ze, zekan1@163.com.

\section{Conflicts of Interest}

The authors have no conflicts of interest.

\section{Authors' Contributions}

Yi Lu, Xiaojie Ding, and Fei Qi have contributed equally to this work.

\section{Acknowledgments}

This work was supported by the National Natural Science Foundation of China (nos. 81704085, 81673866, and 81904214), National Key Research and Development Program of China (no. 2018YFC1705305), Program of Shanghai Academic Leader (no. 20XD1423600), the "Dawn" Program of Shanghai Education Commission (no. 17SG41), Shanghai Municipal Key Clinical Specialty (no. shslczdzk05001), Scientific Research Project of Shanghai Health Committee (no. 20204Y0312), and Three Year Action Plan Project to Promote Clinical Skills and Clinical Innovation Ability of Municipal Hospitals (no. SHDC2020CR4053).

\section{References}

[1] A. J. Boulton, L. Vileikyte, G. Ragnarson-Tennvall, and J. Apelqvist, "The global burden of diabetic foot disease," The Lancet, vol. 366, no. 9498, pp. 1719-1724, 2005.

[2] W. Jeffcoate and K. Bakker, "World diabetes day: footing the bill," The Lancet, vol. 365, no. 9470, 2005.

[3] D. G. Armstrong, A. J. M. Boulton, and S. A. Bus, "Diabetic foot ulcers and their recurrence," The New England Journal of Medicine, vol. 376, no. 24, 2375 pages, Article ID 2367, 2017.

[4] J. Etulain, "Platelets in wound healing and regenerative medicine," Platelets, vol. 29, no. 6, 2018.

[5] L. Kuai, J. T. Zhang, Y. Deng et al., "Sheng-ji Hua-yu formula promotes diabetic wound healing of re-epithelization via activin/follistatin regulation," BMC Complementary and Alternative Medicine, vol. 18, no. 1, p. 32, 2018.

[6] L. Dong, B. Li, and Y. Zhang, "Effect of Shengji Huayu recipe and its disassembled formulae on type I and III collagen synthesis in wound healing fibroblasts," Chinese Journal of Integrated Traditional and Western Medicine, vol. 22, no. 3, pp. 200-202, 2002, in Chinese.

[7] B. Li, Z. Y. Wang, X. L. Xiao, F. L. Li, and B. Fan, "Effects of Shengji Huayu recipe and its decomposed formulas on synthesis of collagen types I and III in granulation tissue of rats in early wound healing," Journal of Chinese Integrative Medicine, vol. 3, no. 3, pp. 216-219, 2005.

[8] Y. F. Wang, X. Li, R. Xu et al., "Effect of Shengji Huayu recipe on the expression of MMP-3 and TIMP-1 in skin ulcer tissue of diabetic rats," Chinese Journal of Integrated Traditional and Western Medicine, vol. 34, no. 2, pp. 218-223, 2014, in Chinese.

[9] K. Takeuchi and K. Amagase, "Roles of cyclooxygenase, prostaglandin E2 and EP receptors in mucosal protection and ulcer healing in the gastrointestinal tract," Current Pharmaceutical Design, vol. 24, no. 18, pp. 2002-2011, 2018.

[10] M. M. Syeda, X. Jing, R. H. Mirza, H. Yu, R. S. Sellers, and Y. Chi, "Prostaglandin transporter modulates wound healing in diabetes by regulating prostaglandin-induced angiogenesis," The American Journal of Pathology, vol. 181, no. 1, 2012.

[11] H. Kampfer, R. Schmidt, G. Geisslinger, J. Pfeilschifter, and S. Frank, "Wound inflammation in diabetic ob/ob mice: functional coupling of prostaglandin biosynthesis to cyclooxygenase-1 activity in diabetes-impaired wound healing," Diabetes, vol. 54, no. 5, pp. 1543-1551, 2005.

[12] Z. Liu, O. Benard, M. M. Syeda, V. L. Schuster, and Y. Chi, "Inhibition of prostaglandin transporter (PGT) promotes perfusion and vascularization and accelerates wound healing in non-diabetic and diabetic rats," PLoS One, vol. 10, no. 7, Article ID e0133615, 2015.

[13] J. Busch, V. Frank, N. Bachmann et al., "Mutations in the prostaglandin transporter SLCO2A1 cause primary hypertrophic osteoarthropathy with digital clubbing," Journal of Investigative Dermatology, vol. 132, no. 10, pp. 2473-2476, 2012.

[14] R. Lu, N. Kanai, Y. Bao, and V. L. Schuster, "Cloning, in vitro expression, and tissue distribution of a human prostaglandin transporter cDNA (hPGT)," Journal of Clinical Investigation, vol. 98, no. 5, pp. 1142-1149, 1996.

[15] Y. Nakamura, T. Nakanishi, H. Shimada et al., "Prostaglandin transporter OATP2A1/SLCO2A1 is essential for body temperature regulation during fever," The Journal of Neuroscience, vol. 38, no. 24, pp. 5584-5595, 2018.

[16] B. Li, Z. Wang, Y. Zhang, X. Xiao, and B. Fan, "Dynamic effects of the method of removing blood stasis and promoting 
muscle growth on the expression of type I and III collagen and MMP-1 mRNA in granulation tissue of experimental wound," Chinese Journal of Integrated Traditional Chinese and Western Medicine, vol. 25, no. 6, pp. 125-128, 2005.

[17] Y. Xiang, X. Li, Q. Xiao et al., "Regulative effects of the method of removing blood stasis and promoting muscle growth on the related genes of MAPK signaling pathway in the repair of diabetic ulcer wounds in SD rats," Journal of Shanghai University of Traditional Chinese Medicine, vol. 28, no. 1, pp. 56-60, 2014.

[18] H. Iwama, S. Amagaya, and Y. Ogihara, "Effect of Shosaikoto, a Japanese and Chinese traditional herbal medicinal mixture, on the mitogenic activity of lipopolysaccharide: a new pharmacological testing method," Journal of Ethnopharmacology, vol. 21, no. 1, pp. 45-53, 1987.

[19] V. L. Schuster, "Prostaglandin transport," Prostaglandins \& Other Lipid Mediators, vol. 68-69, pp. 633-647, 2002.

[20] R. Deng, Y. Qu, and D. Mu, "Prostaglandin transporter," The Chemistry of Life, vol. 29, no. 3, pp. 378-380, 2009.

[21] V. L. Schuster, Y. Chi, and R. Lu, "The prostaglandin transporter: eicosanoid reuptake, control of signaling, and development of high-affinity inhibitors as drug candidates," Transactions of the American Clinical and Climatological Association, vol. 126, pp. 248-257, 2015.

[22] M. M. Syeda, X. Jing, R. H. Mirza, H. Yu, R. S. Sellers, and Y. Chi, "Prostaglandin transporter modulates wound healing in diabetes by regulating prostaglandin-induced angiogenesis," The American Journal of Pathology, vol. 181, no. 1, pp. 334-346, 2012.

[23] K. Bujok, H. Glaeser, W. Schuh et al., "Interplay between the prostaglandin transporter OATP2A1 and prostaglandin E2mediated cellular effects," Cellular Signalling, vol. 27, no. 3, pp. 663-672, 2015.

[24] W. H. Su, M. H. Cheng, W. L. Lee et al., "Nonsteroidal antiinflammatory drugs for wounds: pain relief or excessive scar formation?" Mediators of Inflammation, vol. 2010, Article ID 413238, 8 pages, 2010.

[25] H. Kansu-Celik, O. Gun-Eryılmaz, N. U. Dogan et al., "Prostaglandin E2 induction of labor and cervical ripening for term isolated oligohydramnios in pregnant women with Bishop score $\leq 5$," Journal of the Chinese Medical Association, vol. 80, no. 3, pp. 169-172, 2017.

[26] H. B. Ruan, M. Z. Wang, T. T. Wu, X. Liu, and W. W. Mo, "Clinical effect of bushen quyu recipe combined with acupuncture in treatment of clomiphene-resistant polycystic ovary syndrome infertility patients after cold needle puncture drainage operation," Chinese Journal of Integrated Traditional and Western Medicine, vol. 36, no. 9, pp. 1038-1041, 2016, in Chinese.

[27] X. R. He, Q. C. Lin, and Q. Chen, "Effects of prescription Yiqi Huatan Quyu on oxidative stress level and pathological changes in chronic intermittent hypoxia rat liver," Zhonghua Yi Xue Za Zhi, vol. 97, no. 6, pp. 457-461, 2017, in Chinese.

[28] H.-Z. Liu, X.-X. Han, J. Liu, F.-C. Zhu, and R.-M. Li, "Effect of Taoren Quyu decoction on human endometrial cells and its anti-endometriosis activity in rats," Asian Pacific Journal of Tropical Medicine, vol. 10, no. 7, pp. 696-700, 2017.

[29] P. Yang, F. Qian, M. Zhang et al., "Zishen Tongluo formula ameliorates collagen-induced arthritis in mice by modulation of Th17/Treg balance," Journal of Ethnopharmacology, vol. 250, Article ID 112428, 2020. 